CLINICAL PRACTICE

\title{
A cost-effective strategy for primary prevention of acute rheumatic fever and rheumatic heart disease in children with pharyngitis
}

\author{
J H Irlam, B M Mayosi, M E Engel, T A Gaziano \\ James Irlam is from the Primary Health Care Directorate of the University of Cape Town, South Africa; Prof. Bongani Mayosi and Dr Mark Engel are \\ from the Department of Medicine of the University of Cape Town at Groote Schuur Hospital, South Africa; Dr Thomas Gaziano is a faculty member \\ of the Department of Cardiology, Brigham and Women's Hospital in Boston, USA.
}

Corresponding author: J H Irlam (james.irlam@uct.ac.za)

Primary prevention of acute rheumatic fever (ARF) and rheumatic heart disease (RHD) in children depends on prompt and effective diagnosis and treatment of pharyngitis at the primary level of care. Cost-effectiveness modeling shows that the most cost-effective strategy for primary prevention in South Africa (SA) is to use a simple symptomatic clinical decision rule (CDR) to diagnose pharyngitis in children presenting at the primary level of care and then to treat them with a single dose of intramuscular penicillin. Treat All and CDR2+ strategies are affordable and simple and miss few cases of streptococcal pharyngitis at the primary level of care. The CDR2+ strategy is the most cost-effective for primary prevention of ARF and RHD in urban SA and should complement primordial and secondary prevention efforts.

S Afr Med J 2013;103(12):894-895. DOI:10.7196/SAMJ.7244

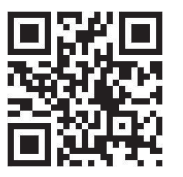

Acute rheumatic fever (ARF) and rheumatic heart disease (RHD) are still a major burden in developing countries. The World Heart Federation has made the elimination of ARF and control of RHD one of the six main goals in its strategic plan through 2015. ${ }^{[1]}$

Primary prevention of ARF and RHD depends on preventing the initial attacks of ARF by means of short-term oral or intramuscular (IM) penicillin treatment of patients presenting with acute sore throat (pharyngitis) caused by an infection with the group A Streptococcus pyogenes. Yet primary prevention has not been widely adopted in developing countries owing to health system barriers and a concern about its cost-effectiveness. ${ }^{[2]}$ Barriers include poor access to primary care, the expense of microbiological diagnosis, shortage of skilled staff and poor public awareness about diagnosis and prompt treatment of suspected streptococcal pharyngitis. A public health strategy of primary prevention in a country like South Africa (SA) should limit costly diagnostic testing, minimise unnecessary antibiotic treatment, and be sensitive enough to minimise missed diagnoses.

The presentation of children with suspected streptococcal pharyngitis to primary care clinics in a RHD study area in Cape Town provided us with the opportunity to undertake a cost-effectiveness analysis of seven strategies for the primary prevention of ARF and RHD in children with pharyngitis in urban SA. ${ }^{[3]}$ We included five single-stage strategies and two two-stage screening strategies in our decision analysis model. Some of these strategies included a simple modified World Health Organization (WHO) clinical decision rule (CDR) that uses a symptomatic score $(0-3)$ based on the presence of enlarged cervical nodes, absence of rhinitis, and absence of rash. ${ }^{[4]}$ The seven categories were thus: $(i)$ empirical treatment with IM penicillin (Treat All); (ii) treatment based on a positive throat culture (Culture All); (iii) treatment based on a symptomatic score of 2 using a modified WHO CDR (CDR 2+); (iv) treatment based on a CDR score of $\geq 3$ (CDR 3+); (v) treating those with a CDR score of $\geq 2$, culturing those with CDR scores $<2$ and then treating positive cultures $(C D R 2+$, Culture CDR negatives); (vi) treating those with a $\mathrm{CDR} \geq 3$, culturing those with $\mathrm{CDR}$ scores $<3$ and treating positive cultures $(C D R 3+$, Culture CDR negatives); and (vii) observation only (Treat None). The two-stage strategies were included to improve the sensitivity of the screening process and to decrease the costs of culture of throat swabs. Rapid streptococcus antigen tests are not currently used in public sector primary care settings and thus were not evaluated. National guidelines on primary prevention encourage IM penicillin because of better adherence and greater effectiveness than oral regimens. ${ }^{[5]}$

Table 1 shows the analysis of the costs (in US\$ at 2010 exchange rates), effects in quality-adjusted life years (QALYs) gained and incremental cost-effectiveness ratios (ICERs) for the seven strategies, in order of increasing cost.

From this analysis it is apparent that:

- Treating all children presenting with suspected pharyngitis with IM penicillin is marginally the least costly strategy (US $\$ 11.19$ or R82 per case).

- There is little difference in the effectiveness of the strategies.

- Treating only children with $\geq 2$ symptoms (CDR2+) on a 3 -symptom score CDR is the most cost-effective strategy (US $\$ 136$ or R994 per QALY gained compared with treating all cases).

- Culturing all children is the most costly strategy. At US\$127 600 or R932 437 per QALY gained, it far exceeds the 2010 costeffectiveness threshold for SA of US $\$ 30000$ per QALY used by the WHO Commission on Macroeconomics and Health.

A group A Streptococcus prevalence of 15\%, an ARF attack rate of $0.3 \%$, and a risk of penicillin-induced anaphylaxis of $1 / 10000$ were deemed appropriate for this setting. In sensitivity analysis testing, only a decrease in group A Streptococcus prevalence below $12.9 \%$, an increase in the ARF attack rate above $1 \%$, and an increase in the risk of penicillin-induced anaphylaxis above 3.4/10 000 cases, altered the model conclusions. 
Table 1. Costs, effects and incremental cost-effectiveness of primary prevention strategies

\begin{tabular}{|c|c|c|c|}
\hline Strategy & Cost (US\$) & Effect (QALY) & ICER (US\$/QALY) \\
\hline Treat all & 11.19 & 22.939936 & \\
\hline CDR 2+ & 11.20 & 22.940002 & 136 \\
\hline CDR $3+$ & 13.00 & 22.939961 & Dominated $^{*}$ \\
\hline Treat none & 14.39 & 22.939899 & Dominated $^{*}$ \\
\hline CDR 2+, Culture CDR negatives & 16.42 & 22.940017 & Dominated $^{+}$ \\
\hline CDR 3+, Culture CDR negatives & 23.89 & 22.940101 & Dominated $^{+}$ \\
\hline Culture All & 27.21 & 22.940128 & 127600 \\
\hline
\end{tabular}

Both the Treat All and CDR2+ strategies are affordable and simple and miss few cases of streptococcal pharyngitis at the primary level of care. The CDR2+ strategy is the most cost-effective in settings with a similar group A Streptococcus prevalence and ARF attack rate in the Cape Town study area. A strategy for primary prevention of ARF and RHD in urban SA should be adopted to complement strategies to improve primordial prevention (better housing and hygiene) and secondary prevention (regular penicillin for those with a history of ARF).

1. Remenyi B, Carapetis J, Wyber R, Taubert K, Mayosi BM. Position statement of the World Heart Federation on the prevention and control of rheumatic heart disease. Nat Rev Cardiol 2013;10(5):284292. [http://dx.doi.org/10.1038/nrcardio.2013.34]
2. Karthikeyan G, Mayosi BM. Is primary prevention of rheumatic fever the missing link in the control of rheumatic heart disease in Africa? Circulation 2009;120(8):709-713. [http://dx.doi.org/10.1161/ CIRCULATIONAHA.108.836510]

3. Irlam J, Mayosi BM, Engel M, Gaziano TA. Primary prevention of acute rheumatic fever and Irlam , Mayosi BM, Engel M, Gaziano TA. Primary prevention of acute rheumatic fever and
rheumatic heart disease with penicillin in South African children with pharyngitis: A cost-

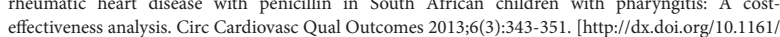
effectiveness analysis. Circ Card

4. Rimoin AW, Hamza HS, Vince A, et al. Evaluation of the WHO clinical decision rule for streptococcal pharyngitis. Arch Dis Child 2005;90(10):1066-1070. [http://dx.doi.org/10.1136/adc.2004.069120]

5. South African National Department of Health. National Guidelines on Primary Prevention and Prophylaxis of Rheumatic Heart Fever and Rheumatic Heart Disease for Health Professionals a Primary Level. Pretoria: DoH, 1997. http://www.kznhealth.gov.za/chrp/documents/Guidelines/ Guidelines\%20National/Rheumatic\%20Heart\%20Disease/Rheumatic\%20heart\%20disease\%20ndoh. pdf (accessed 27 August 2013).

Accepted 11 July 2013. 